\title{
A Large Retrospective Study of 12714 Cases of LEEP Conization Focusing on Cervical Cancer That Colposcopy-Directed Biopsy Failed to Detect
}

\author{
Qing Cong, ${ }^{1,2,3}$ Yu Song, ${ }^{1,2,3}$ Qing Wang, ${ }^{1,2,3}$ Hongwei Zhang $\mathbb{D}^{1,2,3}$ \\ Shujun Gao, ${ }^{1,2,3}$ Ming Du, ${ }^{1,2,3}$ Feng Xie, ${ }^{1,2,3}$ Jing Dong, ${ }^{1,2,3}$ Hua Feng, ${ }^{1,2,3}$ \\ Wenjing Diao, ${ }^{1,2,3}$ Caiying $\mathrm{Zhu},{ }^{1,2,3}$ and Long Sui $\mathbb{D}^{1,2,3}$ \\ ${ }^{1}$ Obstetrics and Gynecology Hospital of Fudan University, Shanghai, China \\ ${ }^{2}$ Shanghai Key Laboratory of Female Reproductive Endocrine Related Diseases, Shanghai, China \\ ${ }^{3}$ Shanghai Medical Center of Key Programs for Female Reproductive Diseases, Shanghai, China
}

Correspondence should be addressed to Long Sui; suilonggyn@163.com

Received 14 November 2017; Accepted 8 February 2018; Published 30 April 2018

Academic Editor: Mittal Suneeta

Copyright (C) 2018 Qing Cong et al. This is an open access article distributed under the Creative Commons Attribution License, which permits unrestricted use, distribution, and reproduction in any medium, provided the original work is properly cited.

Punch biopsy is important in the diagnosis of cervical cancer. However, it may fail to detect early cervical cancers. A retrospective study was performed in the largest academic women's hospital in China to demonstrate cervical cancer that colposcopydirected biopsy failed to detect. Methods. Patients who were diagnosed with high-grade squamous intraepithelial lesion (HSIL), adenocarcinoma in situ (AIS), and persistent low-grade squamous intraepithelial lesion (LSIL) via colposcopy-directed biopsy and had further undergone loop electrosurgical excision procedure (LEEP) conization were included. These procedures were performed at Obstetrics and Gynecology Hospital of Fudan University from July 1, 2013, to December 31, 2016. In total, 5.98\% (760/12714) of patients who underwent conization were diagnosed with invasive cervical cancer. Persistent LSIL (0.24\%), HSIL (6.37\%), and AIS (24.31\%) were detected cancer by conization. Histological subtypes included squamous cell carcinoma (92.0\%), adenocarcinoma (5.1\%), adenosquamous carcinoma (1.8\%), adenoid basal type carcinoma (0.9\%), and small cell neuroendocrine carcinoma (0.1\%). Cytology reports consisted of HSIL (45.4\%), atypical squamous cells of undetermined significance (ASC-US) (16.1\%), and LSIL (11.6\%), and atypical squamous cells cannot exclude HSIL (ASC-H) (9.3\%), squamous cell carcinoma (0.9\%), AGC (atypical glandular cells, $0.9 \%$ ), AIS (0.4\%), and NILM (negative for intraepithelial lesion or malignancy, 15.4\%). The sensitivity of high-risk human papillomavirus (hrHPV) screening (96.4\%) was significantly higher than that of cytology $(84.6 \%)(P<0.01)$, with sensitivity of cotesting at $99.8 \%$ and a ratio of double-negative results at $0.2 \%$. The sensitivity of cytology and hrHPV screening of different cervical cancer histologic subtypes was also demonstrated. In this large retrospective study, we systematically reported the cytology, hrHPV, pathology, and stages of cervical cancer that colposcopy-directed biopsy failed to detect.

\section{Introduction}

Cervical cytology and high-risk human papillomavirus (hrHPV) screening greatly contribute to the early detection of cervical cancer and precancers such as high-grade squamous intraepithelial lesion (HSIL) or cervical intraepithelial neoplasia 2/3 (CIN2/3) [1]. Colposcopy has played a pivotal role in reducing the incidence and mortality from cervical cancer over the past 50 years $[2,3]$. In CIN $2 / 3$ detected by punch biopsy, LEEP conization allows further and more accurate histologic examination of the transformation zone [4]. Although it goes undetected by visual inspection of the naked eye or colposcopy-directed biopsy, unsuspected invasive cancer can be detected by histopathologic examination of conization masses. In conization, loop electrosurgical excision procedure (LEEP) conization, also known as large loop excision of the transformation zone (LLETZ), highfrequency-needles, and laser conization are equally optimal, whereas cold-knife conization is associated with an excessive risk for subsequent obstetric complications [5]. 
Cervical precancers can be treated or even examined for invasive cancers through conization. Treatment management of invasive cervical cancer and its various stages completely differs from that of precancer. Given the wide range of treatment recommendations, accurate diagnosis of cervical precancer and cancer is essential and cervical conization should be given preference over hysterectomy in cases of precancer [6]. Studies have reported that $2.50 \%(1 / 40)-17.39 \%$ $(8 / 46)$ of CIN3 punch biopsies and none $(0 / 94-0 / 24)$ of CIN2 punch biopsies had invasive cancer [4, 7-9]. The ratio of unsuspected, invasive cervical cancer cases to precancerous lesions within these studies differs greatly. Until now, there is no systematic study of these cancers. To gain a deep understanding of these early cervical cancers that failed to be diagnosed by colposcopy-directed biopsy, we retrospectively analyzed 12714 cases of consecutive LEEP conization in the largest Obstetrics and Gynecology Hospital in China.

\section{Materials and Methods}

2.1. Patients. Patients who underwent cervical LEEP conization in Obstetrics and Gynecology Hospital of Fudan University (OGHFU) were included from July 1, 2012, to December 31,2016 . In OGHFU, patients with abnormal cervical cytology or positive hrHPV testing were referred to colposcopy in 2-6 weeks. Colposcopy-directed biopsy was performed on all patients by experienced colposcopists. HSIL, adenocarcinoma in situ (AIS), and low-grade squamous intraepithelial lesion (LSIL) (persistent for 2 or more years or LSIL with cytology of HSIL/atypical squamous cells cannot exclude HSIL [ASC-H]/atypical glandular cells [AGC]/AIS) diagnosed by punch biopsy were subjected to LEEP conization. In addition, one patient with heavy watery vaginal discharge who was diagnosed with cervicitis via punch biopsy also underwent LEEP conization.

2.2. Cytology and hrHPV Testing. In cytology testing, liquid based cytology (ThinPrep [Hologic, Massachusetts, USA] and SurePath [Becton, Dickinson and Company, New Jersey, USA]) were used. In hrHPV testing, the Hybrid Capture 2 (HC2) method (Qiagen, Limburg, Netherlands) was used for the detection of high-risk and intermediate-risk HPV types $16,18,31,33,35,39,45,51,52,56,58,59$, and 68 .

2.3. LEEP Conization and Pathologic Examination. All the procedures were performed by one of 18 staff colposcopists. Different diathermy loops were used depending on the size of cervical lesions to excise and location of the transformation zone. All excisions were performed under colposcopic guidance. The cervical transformation zone and lesion excised to an adequate scale, extending 4 to $5 \mathrm{~mm}$ beyond the lesion in most cases. The tissues were removed to a depth of 7-10 mm, 10-15 mm, and 15-25 mm in type I, II, and III cervical transformation zone, respectively. A second pass with a small loop can also be performed to obtain an endocervical specimen for further histologic evaluation. Information on loop size, volume, length, and thickness of the cone specimen was recorded. For each cone, the pathologists cut the cone tissue into 12 pieces and embedded each piece into a paraffin block. Both ectocervical and endocervical margins were clearly read and reported by pathologists. All pathologic specimens were processed by a standardized protocol, interpreted by an experienced staff pathologist and then verified by another advanced pathologist.

2.4. Statistical Analysis. Approval was obtained from the Institutional Review Board of OGHFU before the data extraction was performed, and all patients gave consent to research. The Pearson chi-square test was used for statistical analysis and conducted using SPSS 16.0 (SPSS Inc., Chicago, Illinois, USA). A $P$ value $<0.05$ was considered statistically significant.

\section{Results}

In total, 12714 consecutive patients of HSIL, AIS, and LSIL diagnosed by colposcopy-directed biopsy underwent LEEP conization. As a result, 5.98\% (760/12714) were further diagnosed with invasive cervical cancer.

In Table 1, the pathology of 759 patients of cervical cancer before and after LEEP conization was shown, excluding one patient who was diagnosed with cervicitis via punch biopsy. By LEEP conization, $0.24 \%$ of LSIL, $6.37 \%$ of HSIL and $24.31 \%$ of AIS diagnosed by punch biopsy were further confirmed as having cervical cancer. Cervical cancer was detected via LEEP cone biopsy in 35 of 144 (24.31\%) patients with AIS in biopsy. Of these 35 patients, 82.9\% (29/35) had adenocarcinoma, $14.3 \%(5 / 35)$ had adenosquamous carcinoma, and $2.8 \%(1 / 35)$ had small cell neuroendocrine carcinoma. The ratio of cervical cancer in LSIL was significantly lower than HSIL $(P<0.01)$. The ratio of cervical cancer in AIS was significantly higher than HSIL $(P<0.01)$.

From Table 2, the mean age of 760 patients was $44 \pm 9$ years (range: 22-70). Five histological subtypes of cervical cancer were detected, including squamous cell carcinoma (92.0\%), adenocarcinoma (5.1\%), adenosquamous carcinoma $(1.8 \%)$, adenoid basal type carcinoma (0.9\%), and small cell neuroendocrine carcinoma $(0.1 \%)$. The mean ratios of stages IA1, IA2, and IB1 were $58.6 \%, 2.4 \%$, and $39.1 \%$, respectively.

Available cytology and hrHPV tests of cervical cancer were shown in Table 3. Diverse cytology was seen in reports of cervical cancer, including HSIL (45.4\%), ASC-US (16.1\%), LSIL (11.6\%), ASC-H (9.3\%), squamous cell carcinoma (SCC, $0.9 \%)$, AGC (0.9\%), AIS (0.4\%), and NILM (negative for intraepithelial lesion or malignancy, 15.4\%). In 259 cases of HSIL, 17 were HSIL (with possibility of cancer) and 2 were HSIL admixed with AGC. In 88 cases of NILM, 82 had available cotesting of hrHPV. These were $98.8 \% \mathrm{hrHPV}$ positive and $1.2 \%$ hrHPV negative. The sensitivity of hrHPV was significantly higher than cytology $(96.4 \%$ versus $84.6 \%$, $P<0.01)$. In 19 cases of negative hrHPV, 18 available cytology reports were $5 \mathrm{HSIL}, 4$ ASC-US, 3 LSIL, 2 ASC-H, 2 AGC, 1 SCC, and 1 NILM. One of $760(0.13 \%)$ patients had doublenegative results of both cytology and hrHPV.

Table 4 showed that 463 cervical cancer cases diagnosed by LEEP conization had both cytology and hrHPV results. Among them, 78.6\% were both cytology and hrHPV positive, 
TABLE 1: Pathology of cervical cancer before and after LEEP conization.

\begin{tabular}{|c|c|c|c|c|c|c|}
\hline \multirow{2}{*}{ Punch biopsy } & \multicolumn{4}{|c|}{ Conization } & \multirow{2}{*}{ Number of LEEP conizations } & \multirow{2}{*}{$\%$ of cervical cancers } \\
\hline & IA1 & IA2 & IB1 & Total & & \\
\hline LSIL & 1 & 0 & 2 & 3 & 1257 & $0.24 \%$ \\
\hline HSIL & 439 & 16 & 266 & 721 & 11312 & $6.37 \%$ \\
\hline AIS & 5 & 2 & 28 & 35 & 144 & $24.31 \%$ \\
\hline Total & 445 & 18 & 296 & 759 & 12713 & $5.97 \%$ \\
\hline
\end{tabular}

Note. The difference between any 2 groups was statistically significant $(P<0.01)$.

TABLE 2: Histological subtypes, ages, and stages of cervical cancer diagnosed by LEEP conization.

\begin{tabular}{|c|c|c|c|c|c|}
\hline $\begin{array}{l}\text { Histological } \\
\text { subtypes }\end{array}$ & $\begin{array}{c}\text { Mean age } \\
\text { (range) }\end{array}$ & $\begin{array}{c}\text { IA1 } \\
n\left(n / \text { total }^{\mathrm{h}}\right)\end{array}$ & $\begin{array}{c}\text { IA2 } \\
n\left(n / \text { total }^{\mathrm{h}}\right)\end{array}$ & $\begin{array}{c}\text { IB1 } \\
n\left(n / \text { total }^{\mathrm{h}}\right)\end{array}$ & $\operatorname{Total}^{\mathrm{h}}$ (n/total) \\
\hline Squamous cell ca & $44(22-70)$ & $438(62.7 \%)$ & $17(2.4 \%)$ & $244(34.9 \%)$ & $699(92.0 \%)$ \\
\hline Adenocarcinoma & $42(29-59)$ & $5(12.8 \%)$ & $1(2.6 \%)$ & $33(84.6 \%)$ & $39(5.1 \%)$ \\
\hline Adenosquamous ca & $42(32-57)$ & $1(7.1 \%)$ & $0(0 \%)$ & $13(92.9 \%)$ & $14(1.8 \%)$ \\
\hline Adenoid basal $\mathrm{ca}^{\mathrm{a}}$ & $63(56-68)$ & $1(14.3 \%)$ & $0(0 \%)$ & $6(85.7 \%)$ & $7(0.9 \%)$ \\
\hline Neuroendocrine ca & $37(37-37)$ & $0(0 \%)$ & $0(0 \%)$ & $1(100 \%)$ & $1(0.1 \%)$ \\
\hline Total & $44(22-70)$ & $445(58.6 \%)$ & $18(2.4 \%)$ & $297(39.1 \%)$ & $760(100 \%)$ \\
\hline
\end{tabular}

${ }^{\mathrm{a}}$ Four of 7 (57.1\%) adenoid basal carcinomas admixed with squamous cell carcinoma. ${ }^{\mathrm{h}}$ Total number of IA1, IA2, and IB1 of the same histological subtype.

TABLE 3: Cytology and hrHPV tests of cervical cancer diagnosed by LEEP conization.

\begin{tabular}{lcc}
\hline Tests & Number of cases & $\%$ \\
\hline Cytology & 570 & $100.0 \%$ \\
HSIL & 259 & $45.4 \%$ \\
ASC-US & 92 & $16.1 \%$ \\
LSIL & 66 & $11.6 \%$ \\
ASC-H & 53 & $9.3 \%$ \\
SCC & 5 & $0.9 \%$ \\
AGC & 5 & $0.9 \%$ \\
AIS & 2 & $0.4 \%$ \\
NILM & 88 & $15.4 \%$ \\
hrHPV & 534 & $100.0 \%$ \\
Positive & 515 & $96.4 \%$ \\
Negative & 19 & $3.6 \%$ \\
\hline
\end{tabular}

TABLE 4: Cotesting results of cervical cancer diagnosed by LEEP conization.

\begin{tabular}{lccc}
\hline Category & hrHPV positive & hrHPV negative & Total \\
\hline Cytology positive & $364(78.6 \%)$ & $17(3.7 \%)$ & $381(82.3 \%)$ \\
Cytology negative & $81(17.5 \%)$ & $1(0.2 \%)$ & $82(17.7 \%)$ \\
Total & $445(96.1 \%)$ & $18(3.9 \%)$ & $463(100.0 \%)$ \\
\hline
\end{tabular}

$17.5 \%$ were only hrHPV positive, $3.7 \%$ were only cytology positive, and $0.2 \%$ were double negative. The sensitivity of cotesting was $99.8 \%$.

In Table 5, the sensitivity of cytology and hrHPV screening for cervical cancer in different histology subtypes was shown. The sensitivity of cytology screening was $85.8 \%$ for squamous cell carcinoma, $65.6 \%$ for adenocarcinoma, $75.0 \%$ for adenosquamous carcinoma, and $80 \%$ for adenoid basal cell carcinoma. The sensitivity of hrHPV screening was $96.9 \%$ for squamous cell carcinoma, 91.2\% for adenocarcinoma, $88.9 \%$ for adenosquamous carcinoma, $100 \%$ for adenoid basal cell carcinoma, and $100.0 \%$ for neuroendocrine carcinoma.

\section{Discussion}

Until now, there were few studies examining how frequently cervical cancer was detected in AIS and HSIL cases diagnosed by punch biopsies. A few earlier studies indicated that $2.50 \%$ $(1 / 40), 2.63 \%(1 / 38), 3.70 \%(2 / 54)$, and $17.39 \%(8 / 46)$ of CIN3 cases were found via conization to have an underlying, unsuspected invasive cancer, respectively [4, 7-9]. Xiang et al. reported $6.74 \%(77 / 1142)$ of HSIL punch biopsies were diagnosed with cancer by conization [10]. Our study reported cervical cancer in $24.31 \%$ (35/144) of punch biopsies detecting AIS and $6.37 \%(721 / 11313)$ of those detecting HSIL, which is the largest retrospective study to date.

In summary, the ratio of cervical cancer confirmed by LEEP conization in AIS was significantly higher than HSIL. In comparison with sufficient inspection of squamous epithelia, which were located on the surface of cervix, most glandular epithelia were in the cervical canal and stroma where crypts were formed. Hence, all glandular epithelia cannot be thoroughly inspected in colposcopy. In addition, punch biopsy and even endocervical curettage could fail to supply enough glandular samples because the early lesions might be in the crypts. Therefore, the difference of histology could be the main reason why a higher rate of adenocarcinoma was detected by excisional procedure.

The ratio of cervical cancer diagnosed by LEEP conization in punch biopsies of LSIL was extremely low $(0.24 \%$, $3 / 1257)$. Among these cases, 1 was diagnosed as microinvasive squamous cell carcinoma with unavailable cytology while the other 2 were diagnosed as invasive squamous cell carcinoma 
TABLE 5: The sensitivity of cytology and hrHPV screening of cervical cancer in different histological subtypes diagnosed by LEEP conization.

\begin{tabular}{|c|c|c|c|c|c|c|}
\hline \multirow{2}{*}{ Histological subtypes } & \multicolumn{3}{|c|}{ Cytology } & \multicolumn{3}{|c|}{ hrHPV } \\
\hline & Number of abnormal cases & Number of tests & Sensitivity & Number of positive cases & Number of tests & Sensitivity \\
\hline Squamous cell ca & 447 & 521 & $85.8 \%$ & 469 & 484 & $96.9 \%$ \\
\hline Adenocarcinoma & 21 & 32 & $65.6 \%$ & 31 & 34 & $91.2 \%$ \\
\hline Adenosquamous ca & 9 & 12 & $75.0 \%$ & 8 & 9 & $88.9 \%$ \\
\hline Adenoid basal cell ca & 4 & 5 & $80.0 \%$ & 6 & 6 & $100.0 \%$ \\
\hline Neuroendocrine ca & NA & 1 & NA & 1 & 1 & $100.0 \%$ \\
\hline Total & 481 & 570 & $84.4 \%$ & 515 & 534 & $96.4 \%$ \\
\hline
\end{tabular}

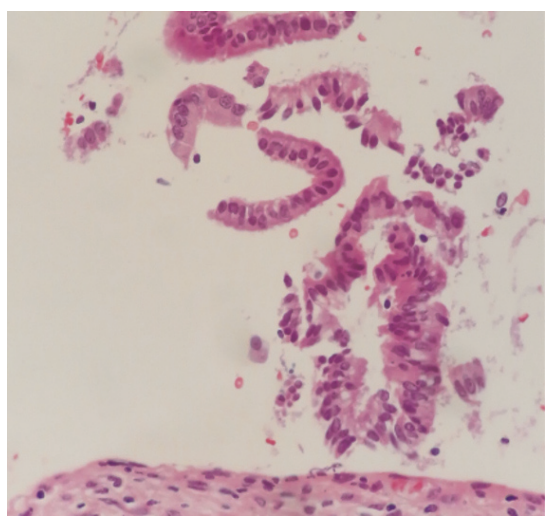

(a)

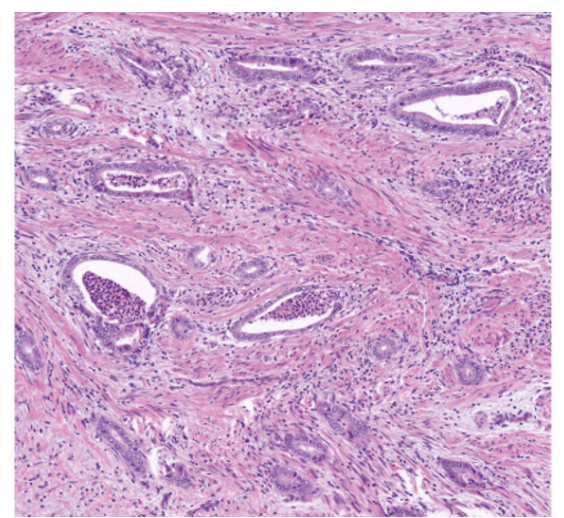

(b)

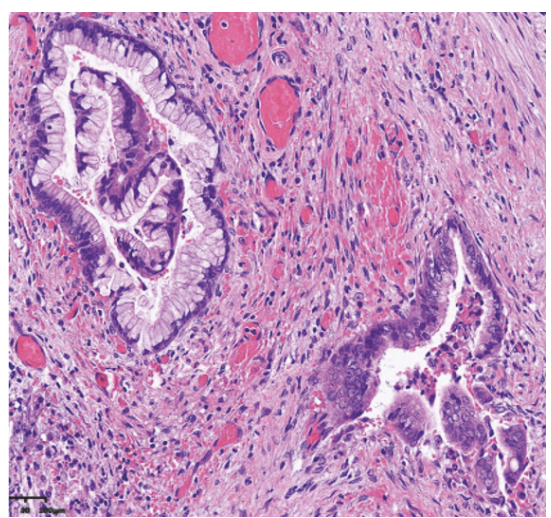

(c)

FIGURE 1: The patient complained of heavy, watery vaginal discharge with negative cytology and hrHPV results. Pathological examination of the punch biopsy and endocervical curettage showed cervicitis (a). Then, histologic examination of the LEEP cone biopsy showed minimal deviation adenocarcinoma (MDA) (b, c).

(IB1) with HSIL cytology. This demonstrates that cancer diagnosis cannot be excluded from HSIL cytology readings and HSILs should be treated by excisional procedures. In fact, a study performed by Kietpeerakool et al. found occult invasive lesions of the cervix in $17 \%$ of women with HSIL Pap smears who underwent a "see and treat" approach [11].

In cervical cancers that punch biopsy failed to detect, squamous cell carcinoma remained the common subtype, followed by adenocarcinoma, adenosquamous cell carcinoma, adenoid basal cell carcinoma, and neuroendocrine carcinoma. Notably, the mean age of adenoid basal cell carcinoma was 63 years, which was significantly greater than the rest of the ages ranging from 37 to 44 years $(P<$ $0.01)$. The ratio of IAl cases to total cases of the same pathology, in descending order, was squamous cell carcinoma (62.7\%), adenoid basal cell (14.3\%), adenocarcinoma $(12.8 \%)$, adenosquamous (7.1\%), and neuroendocrine (0\%). Hence, squamous cell carcinoma is the most common cervical cancer with the majority being IA1.

There were considerable differences among global laboratories in the sensitivity of cytology screening. In the ATHENA (Addressing the Need for Advanced HPV Diagnostics) study, the sensitivity of cytology varied from 42.0 to $73.0 \%$ in CIN grade 2 or worse (CIN2+) [12]. In the cervical cancer screening results among 256,648 women in multiple clinical practices, the sensitivity of cytology was $90.9 \%$ in CIN2/3 and $93.1 \%$ in CIN3 [13]. The sensitivity of hrHPV was
95.8\% in CIN2/3 and $98.8 \%$ in CIN3 [13]. Thus, hrHPV is more sensitive than cytology in precancer screening $(P<$ 0.01 in both CIN2/3 and CIN3). In early cervical cancers that punch biopsy failed to detect, our study showed the sensitivity of cytology and hrHPV was $84.6 \%$ and $96.4 \%$, respectively, which indicated the sensitivity of hrHPV was significantly higher than that of cytology in screening early cervical cancer. In all invasive cervical cancers, the sensitivity of cytology screening $(84.5 \%-89.9 \%)$ [13-15] is similar to the sensitivity of hrHPV screening $(81.4 \%-92.5 \%)$ [14-16]. This demonstrated that the sensitivity of hrHPV decreased as cancer progressed, probably because of the difficulty in detecting hrHPV in necrotic and bleeding tumor samples. Compared with early cervical cancer, the sensitivity of hrHPV screening for all invasive cancers was significantly lower (92.5\% versus $96.4 \%, P=0.01$ ) while the sensitivity of cytology screening was constant $(84.5 \%$ versus $84.6 \%, P=$ 0.98). This suggests that hrHPV screening could help detect more early cervical cancer compared to cytology. In addition, the sensitivities of cytology and hrHPV in different histology subtypes are different from each other. Adenocarcinoma and adenosquamous carcinoma had relatively lower sensitivity of cytology and hrHPV screening compared to other subtypes.

In our study, $0.13 \%(1 / 760)$ of patients had negative cytology and hrHPV results. This patient complained of heavy, watery vaginal discharge. Punch biopsy and endocervical curettage showed cervicitis (Figure 1(a)). Since watery 
vaginal discharge continued to increase, LEEP cone biopsy was performed and the patient was diagnosed with minimal deviation adenocarcinoma (MDA) (Figures 1(b) and 1(c)). Pathologists reviewed punch biopsy and endocervical curettage again and corrected the diagnosis to atypical glands. MDA is rare, consisting of $1-3 \%$ of all cervical adenocarcinomas. MDA is an endocervical adenocarcinoma, which is mucinous and well differentiated. It consists of an endocervical glandular hyperplasia of lobular architecture that resemble glands, but with the characteristics of adenocarcinoma [17]. MDA is associated with the autosomal dominant disease Peutz-Jeghers syndrome (PJS). PJS is characterized by the development of benign hamartomatous polyps in the gastrointestinal tract and hyperpigmented macules on the lips and oral mucosa with mutations in the STK11 gene [18]. MDA is usually hrHPV negative and undetectable by punch biopsy [19]. Hence, in cases of persistent or increasing heavy watery vaginal discharge, cervical conization should be performed to exclude cervical adenocarcinoma, even if cotesting and punch biopsy are normal. Furthermore, in all cervical cancers, Tao et al. reported 3.9\% (9/231) of patients have double-negative results. Compared with squamous cell carcinoma, adenocarcinoma has significantly higher rates of prior negative results with both hrHPV and Pap cytology [14].

\section{Conflicts of Interest}

The authors declare that they have no conflicts of interest.

\section{Authors' Contributions}

Qing Cong and Yu Song contributed equally to the article.

\section{Acknowledgments}

The authors would like to thank Editage (https://www.editage .com) for English language editing. They sincerely appreciate Evans Heather, Shakir Fevzi, Carmel Flynn, and Preeti Chadha from Colposcopy Department of the Royal Free Hospital in the United Kingdom of Great Britain and Northern Ireland for constructive advice and manuscript modification. They thank Xiang Tao and Yihua Sun very much for pathological instruction. Data collection and interpretation were supported by National Natural Science Foundation of China (81471423), Innovation Project of the Science and Technology Commission of Shanghai Municipality (16411950200), and Health Commission Project of Shanghai Municipality (20144Y0098, 201344095).

\section{References}

[1] D. Saslow, D. Solomon, H. W. Lawson et al., "American Cancer Society, American Society for Colposcopy and Cervical Pathology, and American Society for Clinical Pathology screening guidelines for the prevention and early detection of cervical cancer," Journal of Lower Genital Tract Disease, vol. 16, no. 3, pp. 175-204, 2012.

[2] M. J. Khan, C. L. Werner, T. M. Darragh et al., "ASCCP colposcopy standards: role of colposcopy, benefits, potential harms, and terminology for colposcopic practice," Journal of Lower Genital Tract Disease, vol. 21, pp. 223-229, 2017.

[3] E. Surveillance, and End Results Program, https://seer.cancer .gov/statfacts/html/cervix.html.

[4] R. Bonardi, S. Cecchini, G. Grazzini, and S. Ciatto, "Loop electrosurgical excision procedure of the transformation zone and colposcopically directed punch biopsy in the diagnosis of cervical lesions," Obstetrics \& Gynecology, vol. 80, no. 6, pp. 1020-1022, 1992.

[5] K. U. Petry, "Management options for cervical intraepithelial neoplasia," Best Practice \& Research. Clinical Obstetrics \& Gynaecology, vol. 25, pp. 641-651, 2011.

[6] K. E. Lee, C. F. Koh, and W. F. Watt, "Comparison of the grade of CIN in colposcopically directed biopsies with that in outpatient loop electrosurgical excision procedure (LEEP) specimens-a retrospective review," Singapore Medical Journal, vol. 40, no. 11, pp. 694-696, 1999.

[7] G. A. J. McIndoe, M. S. Robson, J. A. Tidy, W. P. Mason, and M. C. Anderson, "Laser excision rather than vaporization: The treatment of choice for cervical intraepithelial neoplasia," Obstetrics \& Gynecology, vol. 74, no. 2, pp. 165-168, 1989.

[8] P. C. Gunasekera, J. H. Phipps, and B. V. Lewis, "Large loop excision of the transformation zone (LLETZ) compared to carbon dioxide laser in the treatment of CIN: a superior mode of treatment," British Journal of Obstetrics and Gynaecology, vol. 97, no. 11, pp. 995-998, 1990.

[9] A. Mandic, S. Knezevic-Usaj, D. Nincic, J. Rajovic, M. Popovic, and T. I. Kapicl, "Comparsion the histopathological findings after cervical biopsy and excisional procedures," Acta Medica, vol. 56, no. 1, pp. 19-22, 2013.

[10] L. Xiang, J. Li, W. Yang et al., "Conization using an electrosurgical knife for cervical intraepithelial neoplasia and microinvasive carcinoma," PLoS ONE, vol. 10, no. 7, Article ID e0131790, 2015.

[11] C. Kietpeerakool, W. Sukkawattananon, J. Srisomboon, S. Khunamornpong, S. Siriaunkgul, and K. Nimmanhaeminda, "Factors predicting occult invasive carcinoma in women undergoing a 'see and treat' approach," Asian Pacific Journal of Cancer Prevention, vol. 9, no. 2, pp. 209-212, 2008.

[12] T. C. Wright Jr., M. H. Stoler, C. M. Behrens, A. Sharma, K. Sharma, and R. Apple, "Interlaboratory variation in the performance of liquid-based cytology: insights from the ATHENA trial," International Journal of Cancer, vol. 134, no. 8, pp. 18351843, 2014.

[13] A. J. Blatt, R. Kennedy, R. D. Luff, R. M. Austin, and D. S. Rabin, "Comparison of cervical cancer screening results among 256,648 women in multiple clinical practices," Cancer Cytopathology, vol. 123, no. 5, pp. 282-288, 2015.

[14] X. Tao, C. C. Griffith, X. Zhou et al., "History of highrisk HPV and Pap test results in a large cohort of patients with invasive cervical carcinoma: Experience from the largest women's hospital in China," Cancer Cytopathology, vol. 123, no. 7, pp. 421-427, 2015.

[15] B. Zheng, Z. Li, C. C. Griffith et al., "Prior high-risk HPV testing and Pap test results for 427 invasive cervical cancers in China's largest CAP-certified laboratory," Cancer Cytopathology, vol. 123, no. 7, pp. 428-434, 2015.

[16] Y. Wu, Y. Chen, L. Li, G. Yu, Y. Zhang, and Y. He, "Associations of high-risk HPV types and viral load with cervical cancer in China," Journal of Clinical Virology, vol. 35, no. 3, pp. 264-269, 2006. 
[17] Y. P. Chen, S. P. Ho, W. S. Liou, and C. J. Chen, "Minimal deviation adenocarcinoma of the uterine cervix," Taiwanese Journal of Obstetrics \& Gynecology, vol. 54, pp. 447-449, 2015.

[18] K. Banno, I. Kisu, M. Yanokura et al., "Hereditary gynecological tumors associated with Peutz-Jeghers syndrome (review)," Oncology Letters, vol. 6, no. 5, pp. 1184-1188, 2013.

[19] A. Molijn, D. Jenkins, W. Chen et al., "The complex relationship between human papillomavirus and cervical adenocarcinoma," International Journal of Cancer, vol. 138, no. 2, pp. 409-416, 2016. 


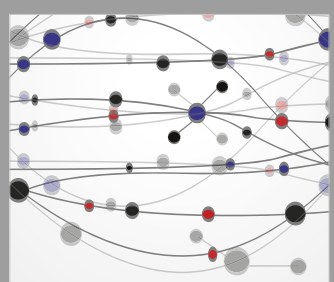

The Scientific World Journal
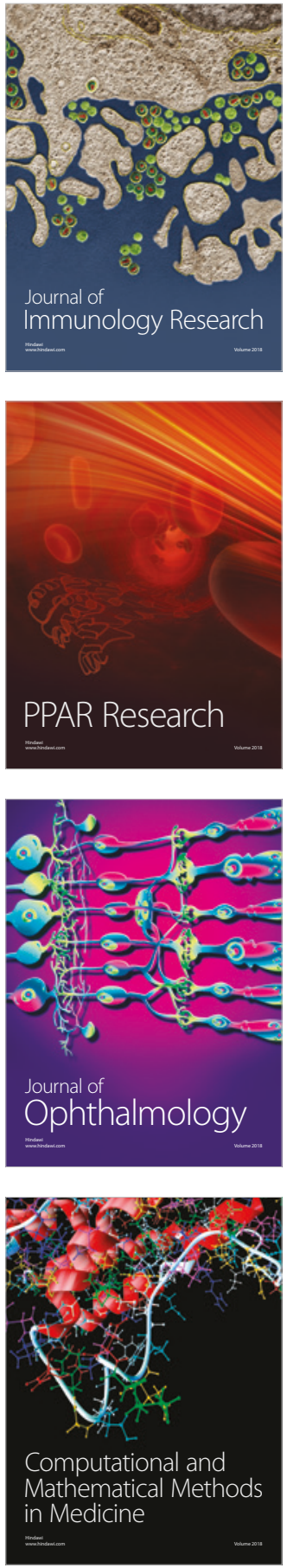

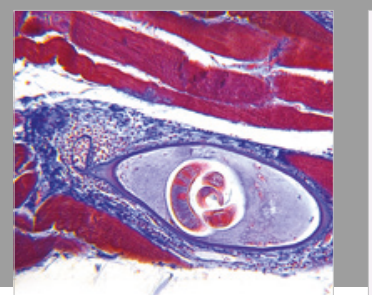

Gastroenterology Research and Practice

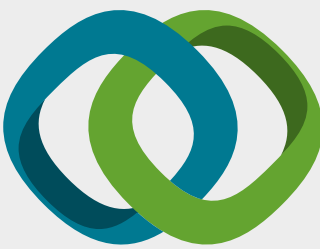

\section{Hindawi}

Submit your manuscripts at

www.hindawi.com
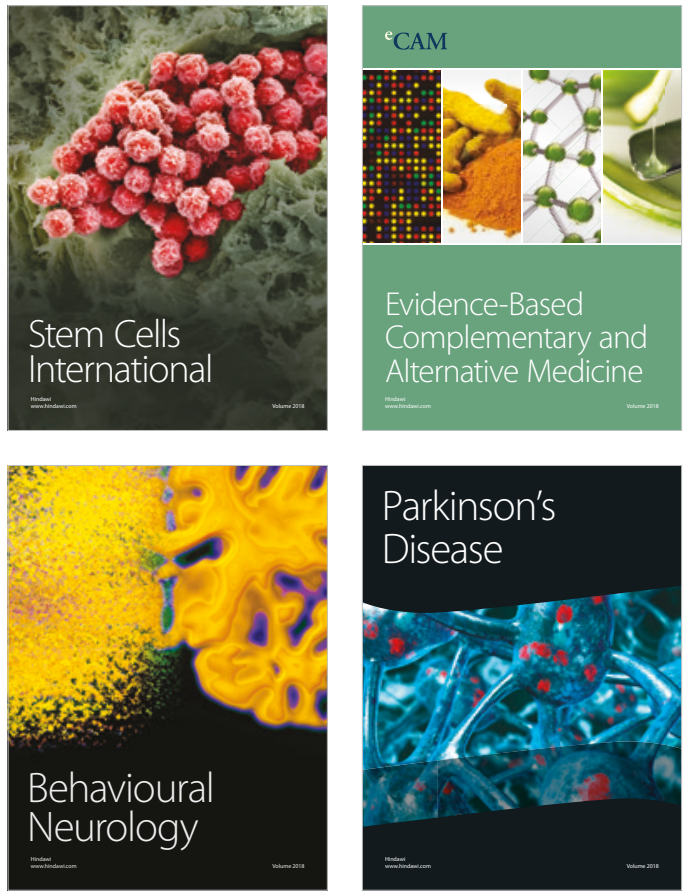

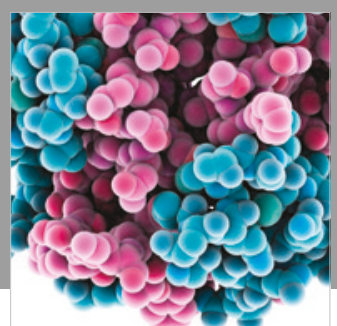

ournal of

Diabetes Research

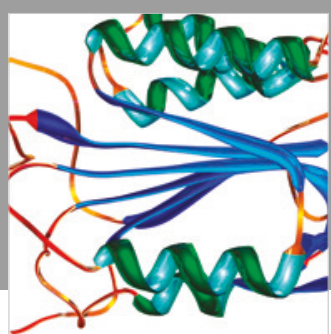

Disease Markers
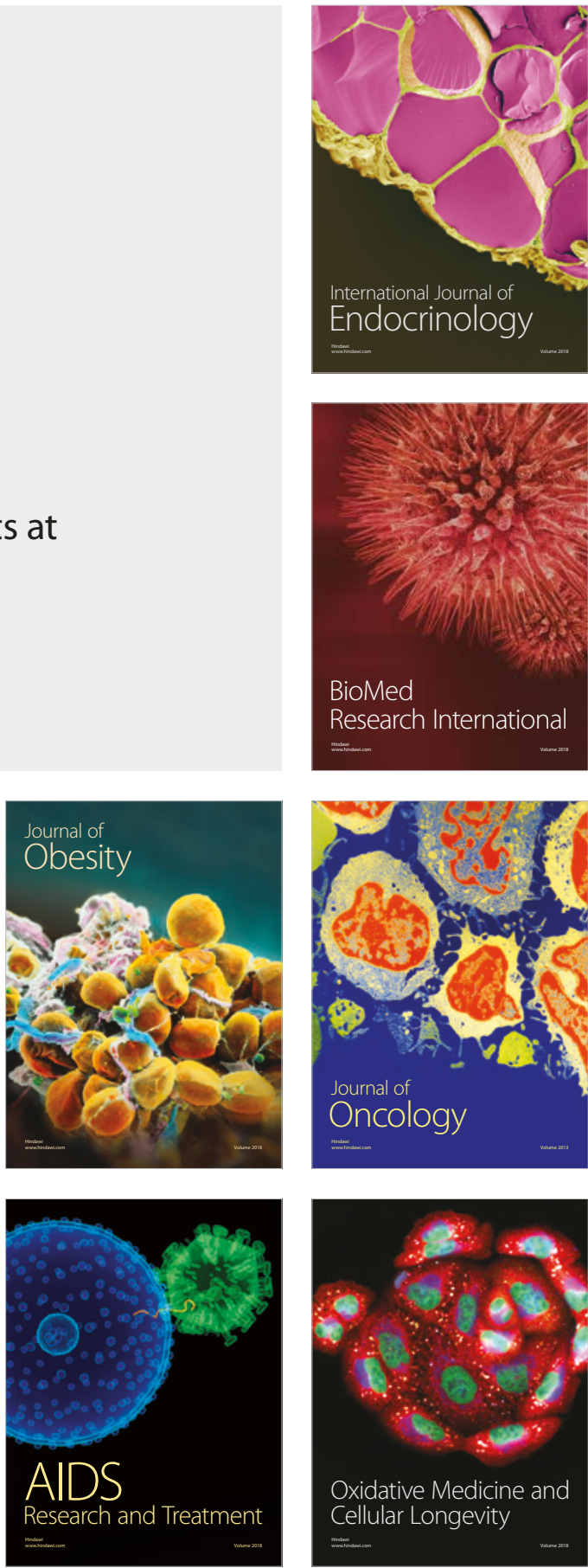